\title{
Experimental Demonstration of 5-Gb/s Polarization-Multiplexed Fiber-Wireless MIMO Systems
}

Zhao, Ying; Pang, Xiaodan; Deng, Lei; Binti Othman, Maisara; Yu, Xianbin; Zheng, Xiaoping; Zhang, Hanyi; Tafur Monroy, Idelfonso

Published in:

2011 Asia-Pacific, MWP/APMP Microwave Photonics, 2011 International Topical Meeting on \& Microwave Photonics Conference

Link to article, DOI:

10.1109/MWP.2011.6088657

Publication date:

2011

Link back to DTU Orbit

Citation (APA):

Zhao, Y., Pang, X., Deng, L., Binti Othman, M., Yu, X., Zheng, X., Zhang, H., \& Tafur Monroy, I. (2011). Experimental Demonstration of 5-Gb/s Polarization-Multiplexed Fiber-Wireless MIMO Systems. In 2011 AsiaPacific, MWP/APMP Microwave Photonics, 2011 International Topical Meeting on \& Microwave Photonics Conference (pp. 13-16). IEEE. https://doi.org/10.1109/MWP.2011.6088657

\section{General rights}

Copyright and moral rights for the publications made accessible in the public portal are retained by the authors and/or other copyright owners and it is a condition of accessing publications that users recognise and abide by the legal requirements associated with these rights.

- Users may download and print one copy of any publication from the public portal for the purpose of private study or research.

- You may not further distribute the material or use it for any profit-making activity or commercial gain

- You may freely distribute the URL identifying the publication in the public portal 


\title{
Experimental Demonstration of 5-Gb/s Polarization- Multiplexed Fiber-Wireless MIMO Systems
}

\author{
Ying Zhao ${ }^{\Uparrow 1,2}$, Xiaodan Pang ${ }^{2}$, Lei Deng ${ }^{2,3}$, Maisara B. Othman ${ }^{2}$, Xianbin Yu ${ }^{\ddagger 2}$, Xiaoping Zheng ${ }^{\Uparrow 11}$, \\ Hanyi Zhang ${ }^{1}$ and Idelfonso T. Monroy ${ }^{2}$ \\ ${ }^{1}$ Department of Electronic Engineering, Tsinghua National Laboratory for Information Science and Technology, \\ Tsinghua University, 100084 Beijing, China. E-mail: 'yinzh@fotonik.dtu.dk, ${ }^{\dagger}$ xpzheng@mail.tsinghua.edu.cn. \\ ${ }^{2}$ DTU Fotonik, Technical University of Denmark, DK-2800, Kgs. Lyngby, Denmark. E-mail: ${ }^{\star}$ xiyu@ $@$ fotonik.dtu.dk. \\ ${ }^{3}$ School of Optoelectronics Science \& Engineering, HuaZhong University of Science \& Technology, Wuhan, China.
}

\begin{abstract}
We experimentally demonstrate a 5-Gb/s fiber-wireless transmission system combining optical polarization-divisionmultiplexing (PDM) and wireless multiple-input, multiple-output (MIMO) spatial multiplexing technologies. The optical-wireless channel throughput is enhanced by achieving a $4 \mathrm{~b} / \mathrm{s} / \mathrm{Hz}$ spectral efficiency. Based on the implementation of constant modulus algorithm (CMA), the $2 \times 2$ MIMO wireless channel is characterized and adaptively equalized for signal demodulation. The performance of the CMA-based channel adaptation is studied and it is revealed that the algorithm is particularly advantageous to the MIMO wireless system due to the interchannel delay insensitivity. The hybrid transmission performance of $26 \mathrm{~km}$ fiber and up to $2 \mathrm{~m}$ wireless MIMO is investigated.
\end{abstract}

Keywords-constant modulus algorithm (CMA); fiber-wireless access; multiple input multiple output (MIMO); polarizationdivision-multiplexing (PDM).

\section{INTRODUCTION}

Scaling wireless channel capacity is important to accommodate the continuing increase in multi-services demand of wireless access systems, and is currently an area of intense research. Multiple-input multiple-output (MIMO) systems have been actively investigated [1] and successfully deployed for current broadband wireless applications such as mobile WiMAX [2]. On the other hand, Radio-over-Fiber (RoF) technology provides elegant solutions for even higher bandwidth requirements beyond $\sim \mathrm{Gb} / \mathrm{s}$ thanks to the ultrawide bandwidth and agility characteristics of photonic devices [3]. To further increase transmission capacity per-wavelength of a fiber-wireless system, polarization-division-multiplexing (PDM) is a promising technique since it offers higher spectral efficiency and transmitter uniformity. Therefore the convergence of multiple antenna and PDM-RoF technologies can be greatly advantageous to emerging high speed telecommunication systems with advantages of high spectral efficiency, flexible transmitter/receiver reconfigurability and efficient wireless channel adaptability [4]

In a PDM-MIMO transmission system, two independent radio signals are carried by two orthogonal polarizations and then sent to an $\mathrm{N} \times \mathrm{N}$ antenna array. The wireless channel capacity can be increased by the factor of $\mathrm{N}$ without additional transmitting power or spectral resources. The key issue for a MIMO system is to adaptively demodulate the received spatial-correlated radio signals in the case of various resolvable and irresolvable wireless paths interfering each other. In general, the synthesized channel can be estimated by using preamble or training symbols known to both transmitter and receiver [5,6], which develops many numerical techniques to perform channel estimation. However, it often needs a large number of overhead symbols to extract the channel response, resulting in the decrease of the net data rate in the system. Furthermore, to obtain preamble or training symbols in the receiver, precise synchronization or timing recovery is essentially necessary $[7,8]$ since preamble-based approaches are all decision-directed. Considering most of synchronization algorithms cannot give a satisfying performance while spatialcorrelation exists in the MIMO case [9], blind channel estimation without resorting to the preamble or training symbols can be very practically promising for MIMO signal demodulation in reality.

The research on hybrid RoF and MIMO systems is still at an early stage with so far limited work on system implementation. Recently, training symbol based MIMO channel estimation with on-off keying (OOK) modulation is reported [10] and a multi-services PDM-RoF system is also demonstrated [4]. The potential of integrating PDM-RoF and MIMO on the system level, in terms of modulation formats, radio frequency (RF) efficiency and algorithm adaptability of channel estimation, remains unexplored.

In this paper, we present and experimentally demonstrate a PDM-RoF system with $1.25 \mathrm{Gbaud} / \mathrm{s}$ QPSK sequence on each polarization state and followed by a $2 \times 2$ MIMO wireless link. At the receiver end, to demodulate the spatial-correlated signals, blind lattice filter adaptation based on constant modulus algorithm (CMA) is implemented in the digital domain. The performance of CMA-based channel estimation is studied and it is validated that MIMO signal demodulation is beneficial from the blind estimation algorithm by avoiding strict channel delay calibration and digital timing recovery. The bit-error-rate (BER) performances of the optical and wireless links are evaluated for the 5-Gb/s QPSK signal at $5.4 \mathrm{GHz} \mathrm{RF}$ carrier over a $26 \mathrm{~km}$ single mode fiber (SMF) plus $2 \mathrm{~m}$ wireless link transmission. The net spectral efficiency of the system is $4 \mathrm{~b} / \mathrm{s} / \mathrm{Hz}$, which is the highest wireless capacity reported at this carrier frequency, to the best of our knowledge. 


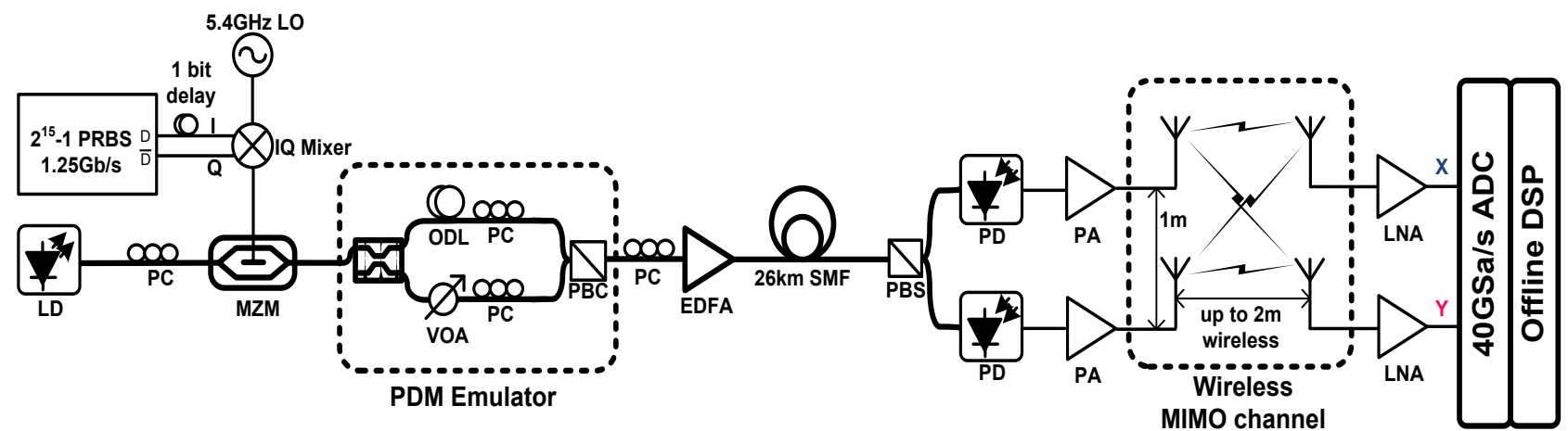

Figure 1. Configuration schematic of the PDM-RoF-MIMO system. LD: laser diode. PC: polarization controller. MZM: Mach-Zehnder modulator. ODL: optical delay line. VOA: variable optical attenuator. PBS: polarization beam splitter. SMF: single mode fiber. PD: photodiode. PA: power amplifier. LNA: low noise amplifier.

\section{EXPERIMENT}

Fig. 1 shows the experimental setup of the PDM-RoFMIMO system. Two polarization-reversed data streams of $1.25-\mathrm{Gb} / \mathrm{s}$ binary pseudo-random bit sequence (PRBS) with a length $2^{15}-1$ are generated. The peak-to-peak voltage of the binary signals is approximately $0.8 \mathrm{~V}$ with minimal waveform overshoots. With 1-bit decorrelation delay introduced to one of the streams, the binary data is mixed with a $5.4 \mathrm{GHz}$ local oscillator in an IQ modulator, forming a $2.5-\mathrm{Gb} / \mathrm{s}$ QPSK stream. The upconverted signal then is modulated onto an optical carrier at $1549.3 \mathrm{~nm}$ at a Mach-Zehnder modulator (MZM). The optical signal is launched into a PDM emulator, in which the optical signal is split into two equal copies, which then are aligned with two orthogonal polarizations and combined by a polarization beam combiner (PBC) to form a PDM-RoF signal with a data rate of $5-\mathrm{Gb} / \mathrm{s}$. In the PDM emulator, the two signals are arbitrarily delayed with respect to each other by an optical delay line to decorrelate two optical copies. The polarization state of the output signal from the PDM emulator is controlled by a primary polarization controller (PC) to roughly adjust the polarization in the transmission fiber. A booster EDFA with a gain of $\sim 20 \mathrm{~dB}$ is cascaded, and then the PDM-RoF QPSK signal is transmitted over a $26 \mathrm{~km}$ single mode fiber (SMF). At the receiver end, the PDM signal is split into two streams onto two orthogonal polarizations (X and $\mathrm{Y}$ ), which are detected and amplified by two photodiodes (PDs) and two power amplifiers (PAs) forming the RF inputs of the wireless MIMO channel. Both of the output radio power after the PAs are measured as $+5 \mathrm{dBm}$.

The wireless channel consists of two identical transmitting antennas and two identical receiving antennas separated by up to $2 \mathrm{~m}$ wireless distance. Two transmitting antennas with $4.4 \mathrm{dBi}$ directional gains are separated by $1 \mathrm{~m}$ height, so as two uni-directional receiving antennas. The link losses of straight channel and cross channel are $20 \mathrm{~dB}$ and $27 \mathrm{~dB}$, respectively, so that $\mathrm{a} \sim 7 \mathrm{dBc}$ lower cross interference is added to the original signal in each receiving antenna.

After undergoing a wireless MIMO channel, the received signals are simultaneously amplified by a low noise amplifier (LNA) and then asynchronously digitized at 40GSa/s using a commercial real-time oscilloscope, with 12-bit analog-todigital converter (ADC) resolution. All results in the receiver side are based on offline processing of at least 2 million

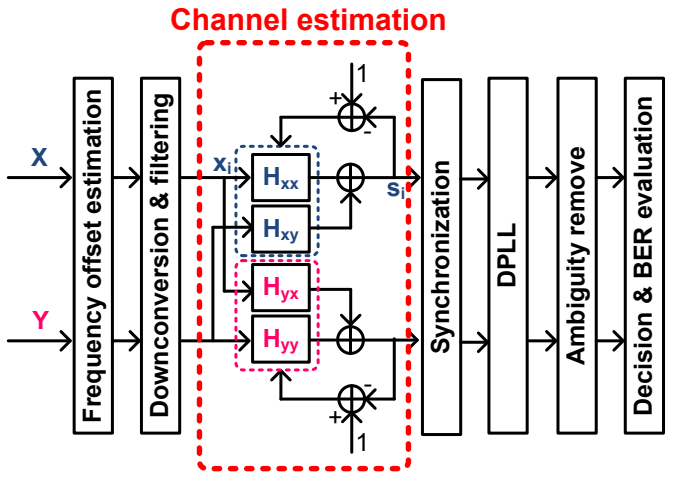

Figure 2. Block diagram of the digital signal processing used in the MIMO signal receiver.

samples on each of the two streams, corresponding to more than 125000 received symbols $(250000$ bits $)$ for bit-error estimation after digital signal processing. As shown in Fig.2, the two sample streams from the ADC are sent to a digital MIMO demodulator for spatial-correlated channel estimation. The first step is a standard implementation of a RF frequency locking based on a non-decision-directed feed forward algorithm in the frequency domain [11]. After frequency estimation, digital local oscillator operating at the estimated frequency is mixed with two streams and lowpass filtering is performed. The filter shape has noticeable impact on the performance of the receiver; Hamming window and Gaussian filters are used in the experiment. The signals are then downsampled to a synchronous $1.25 \mathrm{G}-\mathrm{Sa} / \mathrm{s}$ to perform adaptive MIMO channel estimation. The structure of estimator is based on a lattice filter with transfer functions $\mathrm{H}_{\mathrm{xx}}, \mathrm{H}_{\mathrm{xy}}, \mathrm{H}_{\mathrm{yx}}$ and $\mathrm{H}_{\mathrm{yy}}$ shown in Fig.2, which represents the inverse spatial-correlated matrix of the MIMO channel. Each filter block is implemented in the time domain as a finite impulse response (FIR) filter with optimized number of taps. To realize a preamble-free and channel delay independent estimator, we use the well-proven CMA to perform blind filter adaption [12]. CMA is a simple, robust channel estimation algorithm working independent on training sequence or data decision. In essence, the CMA minimizes the time averaged error

$$
\varepsilon_{C M A}=\overline{1-\left|s_{i}\right|^{2}}
$$

implying the mean distance of equalized symbols $s_{i}$ from the 
constant unit circle in the complex plane. The filter coefficients are adapted according to the algorithm iterations to minimize $\varepsilon_{C M A}$. In our experiment, CMA adaptation works particularly well for spatial-correlated signals with QPSK modulation format. The performance of the algorithm is discussed in section III. After CMA adaptation, the decorrelated output signals are corresponding to two polarizations; the channel impact can be canceled in the blind channel estimator. Subsequently, the symbol timing recovery is achieved by using a cross-correlation based synchronization algorithm. To correct random phase angle rotation of QPSK constellation with respect to the rectilinear desired decision boundaries, a decision-directed digital phase locking loop (DPLL) is implemented on a block of 1000 symbols. In the following, the BER is obtained by direct error counting of 250000bits.

\section{RESUltS AND DisCUSSION}

Fig. 3 shows the constellations of the recovered $5-\mathrm{Gb} / \mathrm{s}$ PDM-RoF signals in both $\mathrm{x}$ and $\mathrm{y}$ polarizations ( $\mathrm{x}-\mathrm{pol}$ and $\mathrm{y}-$ pol) after $1 \mathrm{~m}$ wireless MIMO transmission at $-11 \mathrm{dBm}$ optical power. Without CMA channel estimation, the error vector magnitude (EVM) of constellation clusters are $\sim 30 \%$, which means the QPSK symbols disseminate in a relatively large area due to the cross channel interference. The performance cannot be improved by increasing the transmitting power since the co-increasing interference plays a main role degrading the performance rather than additive noise. Due to the fast timevariant characteristics of the wireless channel, the added interferences fluctuate significantly during the measurement, which results in a requirement of a relatively faster coverage time with respect to the channel fluctuation. The separation between transmitting antennas and receiving antennas also affects the interference level, which shows a closer separation induces lower interference or spatial correlation, vice versa. The small random clusters appearing in the constellation are caused by wireless multipath effect, which corresponds to the various irresolvable paths during the wireless transmission. In the meantime, the digital filter selection also has noticeable effect on the constellation performance. After CMA channel estimation, as shown in the lower row in Fig.3, the constellation clusters dramatically concentrate to smaller clusters. The EVM is $\sim 15 \%$ in this case. The multipath effect plays a main role on cluster dissemination and CMA parameters also determine the convergence performance. From Fig.3, it is also shown that the x-pol signal has a slightly better transmission performance than that of $y$-pol due to the non-identical characteristics between two polarizations.

Another issue noticeable is the necessity of the primary PC in the transmitter. The objective of this PC is to roughly align the two polarizations after fiber transmission with the axis of the PBS in the optical receiver; therefore the polarization dependent channel interference is insignificant. If the primary $\mathrm{PC}$ is removed, with the polarization rotating in the fiber, the interference characteristics vary dramatically and consequently, the channel estimation cannot give an identical performance using same parameters. To keep the performance
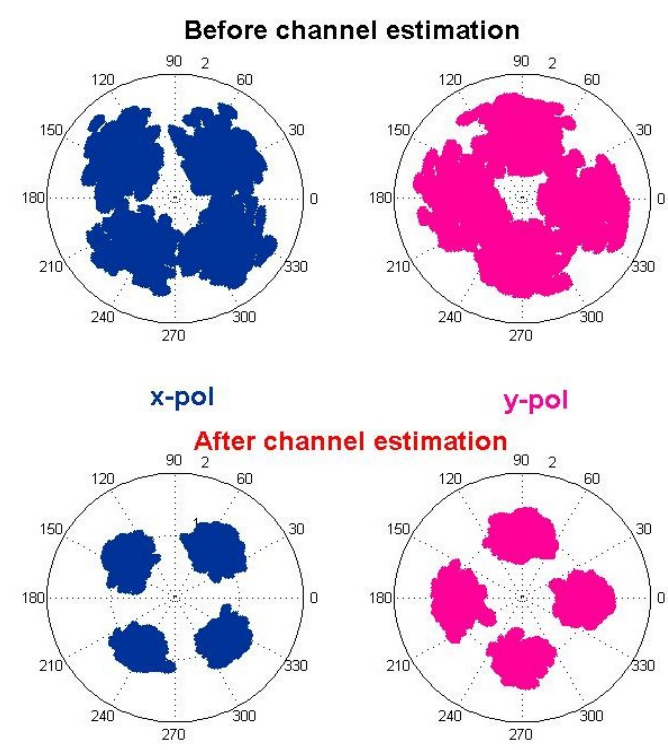

Figure 3. Constellations of the recovered 5-Gb/s x (left column) and y (right column) PDM-RoF signals after $1 \mathrm{~m}$ wireless MIMO transmission without (upper) and with (lower) CMA channel estimation. 125000 symbols.

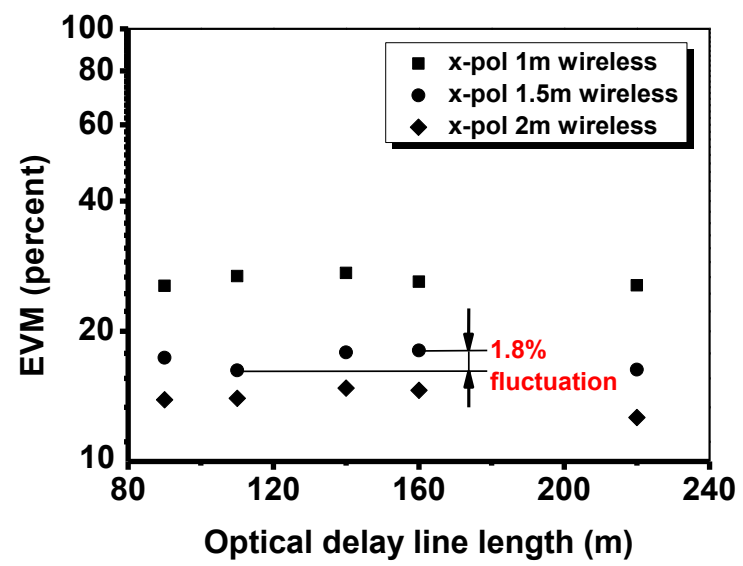

Figure 4. Measured EVM of the demodulated x-polarization QPSK signal versus optical delay between two polarizations with different wireless distances.

of channel estimation stable, the primary PC is preferably kept functional.

Apart from the advantage of non-resorting to preamble or training sequence, the other most significant advantage of using blind CMA adaptation in a MIMO system is it performs consistently with arbitrary change of channel delay. Fig.4 shows the measured EVM of demodulated QPSK signal in the $\mathrm{X}$-polarization against the changing optical delay between two polarizations in the PDM emulator with different wireless transmission distances. A maximal 1.8\% EVM fluctuation indicates the algorithm has a constant performance no matter how much delay is introduced in the optical or the wireless links. This property is not possessed by any decision-directed algorithms [8,11]. 


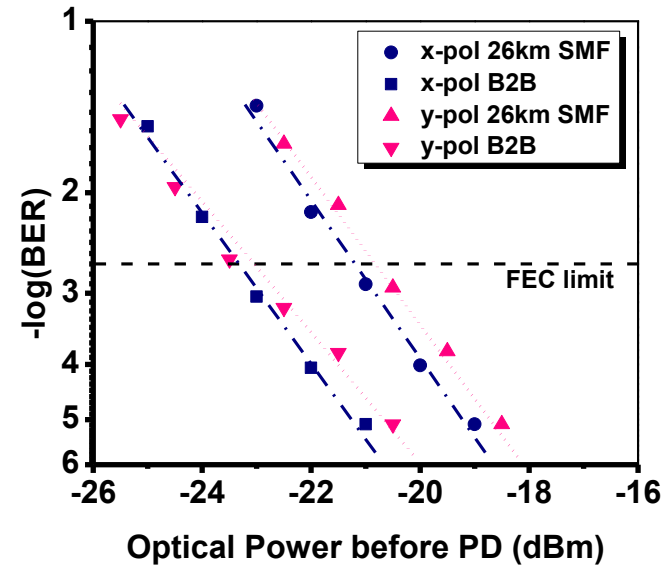

Figure 5. BER performance versus optical power before PD for PDM-RoF signal with $1 \mathrm{~m}$ wireless MIMO transmission.

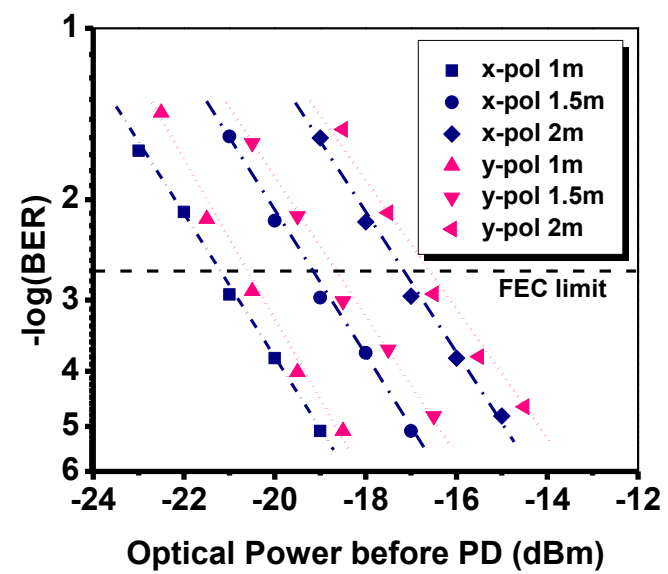

Figure 6. BER performance versus optical power before PD for different wireless transmission distances.

Fig. 5 shows the BER performance as a function of received optical power before the PD in optical B2B and after $26 \mathrm{~km}$ SMF and $1 \mathrm{~m}$ wireless MIMO transmission. The nonidentical channel induced performance difference between the $\mathrm{X}$-pol and y-pol branches is $\sim 0.5 \mathrm{~dB}$. $2 \mathrm{~dB}$ power penalty arisen from fiber dispersion is also observed after $26 \mathrm{~km}$ SMF transmission with respect to the $\mathrm{B} 2 \mathrm{~B}$ case. BER curves for wireless distances of $1 \mathrm{~m}, 1.5 \mathrm{~m}$ and $2 \mathrm{~m}$ after $26 \mathrm{~km}$ fiber transmission are shown in Fig. 6. It indicates $\sim 2 \mathrm{~dB}$ power penalty between $1 \mathrm{~m}$ and $1.5 \mathrm{~m}$ wireless transmission and another $\sim 2 \mathrm{~dB}$ penalty when the distance goes up to $2 \mathrm{~m}$. These penalties are contributed by the increasing cross interference, severer multipath effect and lower signal-to-noise ratio (SNR). Considering the FEC limit at a BER of $2 \times 10^{-3}$, it is clearly shown that in all measured distances we are able to achieve transmissions with BER well below this limit. Another issue noticeable is that when received power goes lower, the transmission system turns to noise-limited, where the CMA adaptation has limited equalization capability. So the transmission performance suffers higher degradation with low SNR.

\section{CONCLUSION}

We have presented and demonstrated the first blind MIMO channel estimation of a PDM-RoF signal at $5-\mathrm{Gb} / \mathrm{s}$. The receiver setup as well as the underlying CMA channel adaptation is described in detail and the algorithm performance is analyzed based on the measured results. The blind algorithm shows an advantage of channel delay insensitivity, which significantly decreases the system complexity. We enlarged the channel throughput at $5.4 \mathrm{GHz}$ RF band by achieving a spectral efficiency of $4 \mathrm{~b} / \mathrm{s} / \mathrm{Hz}$ both in optical and wireless links with PDM and MIMO techniques. Error free PDM-RoF signal over $26 \mathrm{~km}$ fiber and $1 \mathrm{~m}$ wireless transmission yields a required received power of $-18 \mathrm{dBm}$ and $2 \mathrm{~dB}$ power penalty is observed with respect to B2B case. An up to $2 \mathrm{~m}$ wireless MIMO transmission performance is studied showing a required received optical power of $-14 \mathrm{dBm}$. The results show an increased applicability of highly spectral efficient fiber-wireless systems.

\section{ACKNOWLEDGEMENT}

This work was supported in part by National Nature Science Foundation of China (NSFC) under grant No. 60736003, 61025004, 61032005 and National 863 Program under grant No. 2009AA01Z222, 2009AA01Z223.

\section{REFERENCES}

[1] A. J. Paulraj, D. A. Gore, R. U. Nabar, and H. Bolcskei, “An overview of MIMO communications - a key to gigabit wireless," Proc. IEEE, vol. 92, pp. 198-218, Feb. 2004.

[2] W. Fan, A. Ghosh, C. Sankaran, P. Fleming, F. Hsieh, and S. Benes, "Mobile WiMAX systems: performance and evolution," IEEE Commun. Mag., vol. 46, pp. 41-49, Oct. 2008.

[3] J. Yao, "Microwave photonics," IEEE J. Lightw. Technol., vol. 27, pp. 314-335, Feb. 2009.

[4] J. Perez, M. Morant, R. Llorente, and J. Marti, "Joint distribution of polarization-multiplexed UWB and WiMAX radio in PON," IEEE J. Lightw. Technol., vol. 27, pp. 1912-1919, Feb. 2009.

[5] S. L. Jansen, I. Morita, T. C. Schenk, and H. Tanaka, "Long-haul transmission of $16 \times 52.5 \mathrm{Gbits} / \mathrm{s}$ polarization-division-multiplexed OFDM enabled by MIMO processing," J. Opt. Netw., vol. 7, pp. 173182, Feb. 2008.

[6] Y. Han, and G. Li, "Coherent optical communication using polarization multiple-input-multiple-output,” Opt. Expr., vol. 13, pp. 7527-7534, Sep. 2005.

[7] W. Shieh, H. Bao, and Y. Tang, "Coherent optical OFDM: theory and design," Opt. Expr., vol. 16, pp. 841-859, Jan. 2008.

[8] Y. S. Cho, J. Kim, W. Y. Yang, and C. G. Kang, MIMO-OFDM wireless communications with MATLAB. John Wiley \& Sons (Asia), Singapore, 2010.

[9] C. J. Youn, X. Liu, S. Chandrasekhar, Y. Kwon, J. Kim, J. Choe, K. Choi, and E. S. Nam, "An efficient and frequency-offset-tolerant channel estimation and synchronization method for PDM CO-OFDM Transmission," Proc. ECOC'2010, P4.06, Sep. 2010.

[10] S. Fan, H. Chien, A. Chowdhury, C. Liu, W. Jian, Y. Hsueh, and G. Chang, "A novel Radio-over-Fiber system using the xy-MIMO wireless technique for enhanced radio spectral efficiency," Proc. ECOC'2010, Th.9.B.1, Sep. 2010.

[11] H. Meyr, M. Moeneclaey, and S. Fechtel, Digital communication receivers: synchronization, channel estimation, and signal processing. John Wiley \& Sons (NY), USA, 1997.

[12] C. R. Johnson, P. Schniter, T. J. Endres, J. D. Behm, D. R. Brown, and R. A. Casas, "Blind equalization using the constant modulus criterion: A review,” Proc. IEEE, vol. 86, pp. 1927-1950, Oct. 1998. 